\title{
The trans-Neptunian object size distribution at small sizes
}

\author{
R. Gil-Hutton ${ }^{1}$, J. Licandro ${ }^{2}$, N. Pinilla-Alonso ${ }^{3}$, and R. Brunetto ${ }^{4}$ \\ ${ }^{1}$ Complejo Astronómico El Leoncito - CONICET and Universidad Nacional de San Juan, Av. España 1512 sur, \\ J5402DSP - San Juan, Argentina \\ e-mail: rgilhutton@casleo.gov.ar \\ 2 Instituto de Astrofísica de Canarias, c/vía Láctea s/n, E38205, La Laguna, Tenerife, Spain \\ 3 Fundación Galileo Galilei \& Telescopio Nazionale Galileo, PO Box 565, 38700, S/C de La Palma, Tenerife, Spain \\ 4 Institut d'Astrophysique Spatiale, Université Paris-Sud, bâtiment 121, 91405 Orsay Cedex, France
}

Received 6 November 2008 / Accepted 4 March 2009

\section{ABSTRACT}

\begin{abstract}
Aims. The aim of this work is to estimate the size distribution of small Trans-Neptunian objects.
Methods. We simulate the irradiation and collisional processes affecting the surface of a Trans-Neptunian object for the first time using as a constraint the peculiar crystalline to amorphous water ice ratio observed on (136108) $2003 E L_{61}$.

Results. We find that the size distribution changes its exponent from $q_{0}=4.2$ at larger sizes to $q_{1}=2.40 \pm 0.3$ at the smaller ones, with a break radius of $r_{1}=35 \pm 15 \mathrm{~km}$. If this size distribution is applied to studying the collisional surface evolution of (136108) $2003 E L_{61}$, we find that the object must be covered by a thin ice crust of $\approx 0.12 \mathrm{~cm}$, while the original composition of the object is still present at $1.61 \mathrm{~cm}$ or more below the surface. This result is not affected by a collision with a large projectile that occurred by chance more than $10^{9} \mathrm{yr}$ ago since after a short time the mean value obtained for the crystalline to amorphous water ice ratio is indistinguishable from the one obtained without a collision with a large projectile. Since the simulations are not sensitive to the effects of very small projectiles $\left(r_{\mathrm{p}}<10-30 \mathrm{~m}\right)$, it is possible that the exponent of the size distribution for these very small objects changes again, approaching a Donhanyi’s size distribution.
\end{abstract}

Key words. Kuiper Belt - solar system: formation - astrochemistry - methods: numerical

\section{Introduction}

The Trans-Neptunian belt $(\mathrm{TNb})$ is a vast swarm of icy bodies beyond the orbit of Neptune in the outer Solar System. Over the past decade, a great deal of effort has been invested in the study of the Trans-Neptunian objects (TNOs) since they can provide essential information about the conditions in the early Solar System environment. Even though the uppermost surface layer of these bodies could be affected by solar radiation, cosmic rays, interplanetary dust, and collisions, they represent the most pristine material available to study in the Solar System.

The physical properties of TNOs are indicative of the accretion process, and particularly its size distribution may hold clues to the process of giant planet formation (Davis \& Farinella 1996; Gladman et al. 2001; Kenyon \& Bromley 2004). Because of TNOs faintness, the size distribution of the population is well-determined observationally only for objects with radius $r$ greater than $\sim 50 \mathrm{~km}$, and it is usually represented by a power law $\mathrm{d} N(>r) \propto r^{-q}$ with $q=4.0-4.8$ (Trujillo et al. 2001a,b; Petit et al. 2006). It is important to note that this distribution must change at some break radius $r_{\mathrm{b}}$ to avoid a divergence in the mass or reflected surface brightness of the TNb population (Kenyon \& Windhorst 2001), but a reliable value for this parameter is not known and theoretical predictions are uncertain (Kenyon \& Bromley 2004; Pan \& Sari 2005). The first observational constraint has come from Bernstein et al. (2004), who found only 3 TNOs at $m_{\mathrm{R}} \sim 27-28(r \sim 23-12 \mathrm{~km}$ for an albedo of $\approx 0.04)$ where they expected more than 80 bodies based on an extrapolation of the size distribution of Trujillo et al.. This deficit in small TNOs indicates a break in the size distribution, but it is inconsistent with the previously expected small-end distribution with $q=3.5$ due to the effects of destructive collisions (Davis \& Farinella 1996; Kenyon 2002). Later, Fraser et al. (2008) found no evidence of a slope change in the size distribution for objects brighter than $m_{\mathrm{R}} \sim 24.3$, but Fuentes \& Holman (2008), Fraser \& Kavelaars (2008), and Fraser \& Kavelaars (2009) confirm the broken power-law size distribution.

An interesting possibility for testing the size distribution of TNOs at small sizes is to study the collisional effects of projectiles on the surface of large TNOs. Spectroscopic and spectrophotometric studies show that about $70 \%$ of TNOs present a surface enriched in complex organics (Brunetto et al. 2006). Long-term processing by high-energy particles and solar radiation on icy bodies induces the formation of organic species in their outer layers, resulting in a mantle that covers the unprocessed original ices (Moore et al. 1983; Johnson et al. 1984; Strazzulla \& Johnson 1991). A few tens of micrometers of an organic-rich layer are already able to mask the presence of water and other volatiles below the surface spectroscopically in the near-IR (Brunetto \& Roush 2008). It has been shown that several TNOs have surfaces covered by fresh water ice, with spectra that are neutral and featureless in the visible and show strong water ice absorption bands in the near infrared (Brown et al. 1999; Licandro et al. 2006; Barkume et al. 2006; Trujillo et al. 2007; Brown et al. 2007; Pinilla-Alonso et al. 2007).

Among these TNOs with almost pure water ice on their surfaces, (136108) $2003 E L_{61}$ is the fourth largest known TNO and it has a $\approx 1: 1$ intimate mixture of crystalline and amorphous water ice as the most probable composition of its surface, constraining the presence of other minor constituents to a maximum 
of $8 \%$ (Pinilla-Alonso et al. 2009). These authors showed that cryovolcanism is not responsible for the crystalline water ice observed on the surface of (136108) $2003 E L_{61}$ since they did not find evidence in the spectrum of the ammonia hydrate absorption band at $2.2 \mu \mathrm{m}$, which is an expected product of this mechanism. On the other hand, the process proposed by Zheng et al. (2008) where the thermal recrystallization could dominate the irradiation amorphization for temperatures $>40-50 \mathrm{~K}$ is not valid here since a mean surface temperature of $\sim 30 \mathrm{~K}$ is obtained for this object (Merlin et al. 2007) using the Stefan - Boltzmann law and the albedo determined by Rabinowitz et al. (2006).

Then, following the suggestion of Licandro et al. (2006) to explain the presence of crystalline water on (55636) $2002 T X_{300}$, Pinilla-Alonso et al. proposed a scenario where the resurfacing is produced by collisions. A collisional event releases energy that could be partially converted into heat that would produce the crystallization of water ice, but the eroded material would also be sublimated and distributed homogeneously over the surface of the TNO while recondensing. After the collisional event, the irradiation process starts to transform crystalline into amorphous water ice again (Kouchi \& Kuroda 1990; Moore \& Hudson 1992; Strazzulla et al. 1992; Leto \& Baratta 2003), while the final crystalline to amorphous ice ratio depends on the collisional and irradiation timescales and on the size distribution of the projectile population.

In this paper we present results about the best fit to the TNO size distribution at small sizes and its break radius, using the peculiar crystalline to amorphous water ice ratio observed for (136108) $2003 E L_{61}$ as constraint. In Sect. 2 we describe the model used to simulate the collisional and irradiation processes, and in Sects. 3 and 4 we present and discuss the results, respectively. Finally, in Sect. 5 we summarize the conclusions.

\section{The model}

The model used to simulate the evolution of the surface of (136108) $2003 E L_{61}$ is very simple. We assume that the target has a radius $r_{\mathrm{t}}$ and that it is composed of a mix of rock and water ice with mean density $\rho_{\mathrm{t}}=\rho_{\mathrm{s}} \times\left(1-f_{\text {ice }}\right)+\rho_{\text {ice }} \times f_{\text {ice }}$, where $\rho_{\mathrm{s}}$ and $\rho_{\text {ice }}$ are the rock and ice densities, respectively, and $f_{\text {ice }}$ is the ice fraction in the original composition. Any non-disruptive collision between a projectile, with radius $r_{\mathrm{p}}$ and density $\rho_{\mathrm{p}}$, and the target produces a hemispherical crater with diameter

$D_{\text {cra }}=2.52 r_{\mathrm{p}}\left(\beta \frac{\rho_{\mathrm{p}}}{\rho_{\mathrm{t}}}\right)^{\frac{1}{3}}\left(\frac{3.22 g r_{\mathrm{p}}}{v_{\text {col }}^{2}}\right)^{\frac{-\alpha}{3}}$,

where $g$ is the surface gravity of the target, $v_{\text {col }}$ the collision velocity, $\alpha$ and $\beta$ constants dependent upon the mechanical properties of the target material, and the equation must be evaluated in cgs units (Holsapple 1993). Typical values of the constants for water ice are $\alpha=0.65$ and $\beta=0.2$. Assuming a crater depth/diameter ratio $\mu=h_{\text {cra }} / D_{\text {cra }}=0.18-0.20$, which is the value for simple craters on the Moon and icy Galilean satellites (Schenk et al. 2004), the water ice mass excavated from the crater is

$M_{\text {ice }}=\left(V_{0}+V_{1} f_{\text {ice }}\right) \rho_{\text {ice }}$,

where $V_{0}$ is the volume of pure ice in the crater, deposited on the surface by previous collisions and forming a layer of thickness $h_{\text {ice: }}$ :

$V_{0}=\left[\begin{array}{ll}\frac{\pi}{24 \mu^{2}}\left(3+4 \mu^{2}\right)\left[h_{\mathrm{cra}}^{3}-\left(h_{\mathrm{cra}}-h_{\mathrm{ice}}\right)^{3}\right] & , \text { for } h_{\text {ice }}<h_{\mathrm{cra}}, \\ \frac{\pi}{24 \mu^{2}}\left(3+4 \mu^{2}\right) h_{\mathrm{cra}}^{3} & , \text { for } h_{\text {ice }} \geq h_{\mathrm{cra}},\end{array}\right.$ and $V_{1}$ is the volume of mixed ice in the crater, excavated at depths greater than $h_{\text {mix }}$ where it is possible to find material with the original composition of the target:

$$
V_{1}=\left[\begin{array}{ll}
\frac{\pi}{24 \mu^{2}}\left(3+4 \mu^{2}\right)\left(h_{\mathrm{cra}}-h_{\mathrm{mix}}\right)^{3} & , \text { for } h_{\mathrm{mix}}>h_{\mathrm{cra}} \\
0 & , \text { for } h_{\mathrm{mix}} \leq h_{\mathrm{cra}} .
\end{array}\right.
$$

The total kinetic energy produced by a collision with a projectile of mass $m_{\mathrm{p}}$ is $E_{\mathrm{k}}=m_{\mathrm{p}} v_{\text {col }}^{2} / 2$, which transforms into other kinds of energy: fractions $\delta_{\mathrm{k}}$ and $\delta_{\mathrm{T}}$ of the total kinetic energy transform into kinetic energy of the fragments produced by the collision and thermal energy, respectively, while the remaining fraction is used in mechanical work. Typical values for these parameters are $\delta_{\mathrm{k}}=0.1-0.2$ (Davis \& Farinella 1997; Gil-Hutton 1997) and $\delta_{\mathrm{T}}=0.1$ (Orosei et al. 2001). Then, the water ice mass sublimated in the collision is $M_{\mathrm{sub}}=\delta_{\mathrm{T}} E_{\mathrm{k}} / H$, where $H=2.778 \times 10^{10} \mathrm{erg} \mathrm{g}^{-1}$ is the latent heat of ice sublimation (Skorov et al. 2001). In the case where $M_{\text {sub }}>M_{\text {ice }}$ all the water ice mass in the crater is sublimated and the remaining thermal energy not used in this process goes to heating other material.

Since the timescale to transport vapor globally is short compared with the radiative cooling timescale of the vapor, the ice mass sublimated in the collision and retained by the target should form transient atmospheres lasting tens of hours to a few days and finally precipitating again on the target surface (Stern 2002), forming a crystalline water ice layer of thickness $h_{\text {dep }}=M_{\text {sub }} /\left(4 \pi \rho_{\text {ice }} r_{\mathrm{t}}^{2}\right)$. Since we are looking for a global effect on the target surface, we only take into account collisions producing a crystalline water ice layer with $h_{\text {dep }} \geq 0.01 \mu \mathrm{m}$. This thickness is less than the expected water ice particle size formed as a loose aggregate $(10-50 \mu \mathrm{m}$, Sunshine et al. 2006), but in this way we obtain some compensation for global ice layers created by the ice accumulation due to the large number of small projectile collisions during each time step of the integration.

The mean number of collisions a target with radius $r_{\mathrm{t}}$ receive in a time interval $\Delta t$ is

$\left\langle N_{\text {col }}\right\rangle=\left\langle P_{\mathrm{i}}\right\rangle \tau^{2} \Delta t N_{\text {pro }}\left(>r_{\mathrm{p}}\right)$,

where $\left\langle P_{i}\right\rangle$ is the mean intrinsic collisional probability of the target, $\tau=\left(r_{\mathrm{t}}+r_{\mathrm{p}}\right)^{2}$ the geometric cross-section, and $N_{\text {pro }}\left(>r_{\mathrm{p}}\right)$ is the number of projectiles with radius larger than $r_{\mathrm{p}}$. To find a value for $N_{\text {pro }}\left(>r_{\mathrm{p}}\right)$, it is necessary to know the cumulative size distribution of the real projectile population, i. e. the size distribution of the $\mathrm{TNb}$. We model the size distribution by a power law of the form $\mathrm{d} N_{\text {pro }}(>r) \propto r^{-q} \mathrm{~d} r$, where $q$ is a characteristic exponent. Considering that the size distribution could change for different size ranges, the number of projectiles with radius greater than $r_{\mathrm{p}}$ is

$N_{\text {pro }}\left(>r_{\mathrm{p}}\right)=K_{0} \int_{r_{1}}^{r_{0}} r^{-q_{0}} \mathrm{~d} r+K_{1} \int_{r_{2}}^{r_{1}} r^{-q_{1}} \mathrm{~d} r$

where $K_{0}$, and $K_{1}$ are constants, $r_{0}$ the largest object in the population, and $r_{1}$ the break radius of the size distribution for two arbitrary size ranges. Since (136108) $2003 E L_{61}$ is a classical TNO, as first guest we used the size distribution proposed by Trujillo et al. (2001b) for large classical objects, characterized by an exponent $q_{0}=4.2$ and an estimation of $4.7 \times 10^{4}$ objects with $r>50 \mathrm{~km}$, a break radius in the range $r_{1}=20-50 \mathrm{~km}$, and size distributions for the smaller objects with exponents ranging from $q_{1}=3.5$ (Dohnanyi 1969) to $q_{1}=2.0$.

On the other hand, the irradiation of water ice by cosmic rays or UV radiation converts crystalline water ice into amorphous water ice. Leto \& Baratta (2003) find from laboratory experiments that a complete amorphization is obtained by irradiating 
crystalline water ice with a dose of at least $10 \mathrm{eV} /$ molecule and that the process is roughly linear with the dose. Then, taking the results of Strazzulla et al. (2003) about the time needed to produce a radyolitic change in irradiated water ice into account, we can infer a timescale for this process of about $10^{9} \mathrm{yr}$ at a heliocentric distance of $40 \mathrm{AU}$ and its effects are also linear with time.

To apply this model to (136108) $2003 E L_{61}$ we need some physical constraints and values for different parameters related with the target and the projectile population. Since the crystalline to amorphous water ice ratio for this object was obtained from visible-NIR reflectance spectroscopy, and such observations only retrieve information from the most external surface layers of the body, we performed several tests to estimate the thickness of the water ice layer that is able to mask any spectral signature of the underlying material.

We used a multi-layer scattering model, as developed and described by Brunetto \& Roush (2008). The model to calculate the reflectance from a system where a layer overlies an infinitely thick substrate is based on Eqs. (7.45c), (8.89), (9.14), and (11.24) from Hapke (1993). Assuming that the material under the water ice layer is mainly composed of silicates, in Fig. 1 we show our results for an ice layer (grain size of $10 \mu \mathrm{m}$ ) with an amorphous to crystalline water ratio similar to that observed on (136108) $2003 E L_{61}$ (Pinilla-Alonso et al. 2009). The optical constants used for water ice are the same as in PinillaAlonso et al., whereas for silicates (olivine and pyroxene) we used the optical constants by Brunetto et al. (2007), assuming a reasonable grain size of $20 \mu \mathrm{m}$. We found that an ice layer with thickness $\approx 200 \mu \mathrm{m}$ is enough to mask any silicate contribution to the spectrum. Thus, the numerical model described below takes spectral information coming from a layer with thickness $h_{\text {spec }} \leq 200 \mu \mathrm{m}$ into account.

One important parameter for evaluating the collisional evolution is the mean intrinsic collision probability, which can be inferred from statistical studies of the occurrence of orbital encounters between the target and the projectile population using the method developed by Marzari et al. (1996). In this method the target and a projectile population were numerically integrated during a time span $T_{\text {int }}$ and the encounter distance and encounter velocity between the target and any projectile were recorded. Since Marzari et al. shown that the distribution of the cumulative number of encounters for an encounter distance less than $d_{\mathrm{enc}}$ is proportional to $d_{\mathrm{enc}}^{2}$, a distribution of the form

$N_{\text {enc }}\left(<d_{\text {enc }}\right)=P_{1} \times d_{\text {enc }}^{2}$

was assumed. The proportionally constant $P_{1}$ is found by a fit to the data, taken as standard deviation for each point $\sqrt{N_{\text {enc }}}$. Then, the mean intrinsic collision probability is obtained from

$\left\langle P_{i}\right\rangle=\frac{P_{1}}{n_{\text {pair }} T_{\mathrm{int}}}$,

where $n_{\text {pair }}$ is the number of different pair of objects which can be formed within the interacting population. Since in this case the $\mathrm{TNb}$ is the main source of projectiles, it is enough to use as the projectile population a sample of particles with the same orbital element distribution than that observed for the TNOs. This sample of 1173 objects was obtained from the ASTORB database (ftp://ftp. lowell.edu/pub/elgb/ astorb . html). The hybrid integrator EVORB (Fernández et al. 2002) was used for the numerical integration of the targets and the particles of the projectile population, under the gravitational influence of the Sun and the planets from Mercury to Neptune.

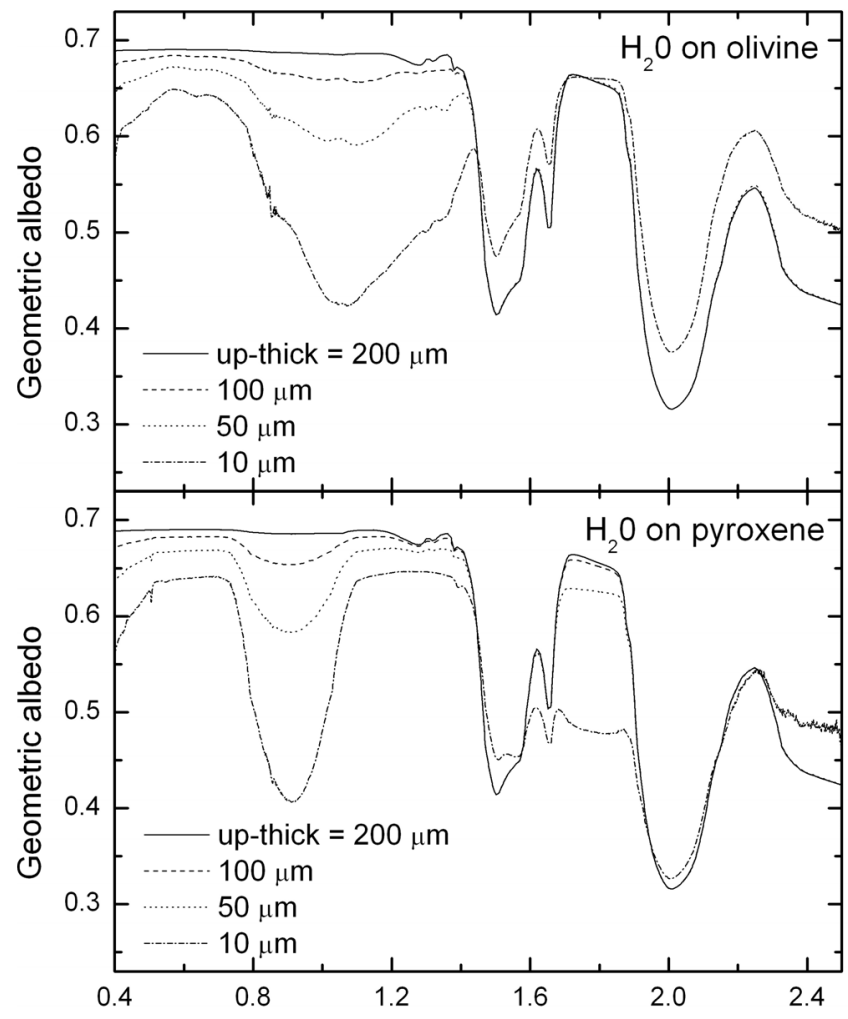

Fig. 1. Synthetic spectra obtained using a two-layer model (Brunetto \& Roush 2008). Upper layer is composed of water ice (grain size of $10 \mu \mathrm{m})$ with amorphous to crystalline water ratio similar to what is observed on (136108) $2003 E L_{61}$ (Pinilla-Alonso et al. 2009). Lower layer is composed of olivine (upper panel) or pyroxene (lower panel), with grain size of $20 \mu \mathrm{m}$. Four models for different upper layer thickness (10, 50,100 , and $200 \mu \mathrm{m}$ ) are shown, to find the minimum value required to mask any spectral contribution of underlying silicates.

The integration was made during a time span of $T_{\mathrm{int}}=3 \times 10^{6} \mathrm{yr}$, and an encounter was recorded every time the mutual distance between the target and a particle was less than 0.1 AU. As a sub-product, the method of Marzari et al. (1996) also provide the collision velocity distribution and the mean collision velocity, $\left\langle V_{\text {col }}\right\rangle$, for the target. The values obtained for (136108) $2003 E L_{61}$ are $\left\langle P_{i}\right\rangle=1.47 \pm 0.02 \times 10^{-22} \mathrm{~km}^{-2} \mathrm{yr}^{-1}$ and $\left\langle V_{\text {col }}\right\rangle=2.48 \pm 0.86 \mathrm{~km} \mathrm{~s}^{-1}$. These values are different from the mean collisional velocity and mean intrinsic collisional probability found by Dell'Oro et al. (2001) for the TNOs, but it is a consequence of the excited orbit of this object in comparison with the mean orbit of this population. The projectile collision velocities for the simulation were taken from the collision velocity distribution found for this object, which is shown in Fig. 2.

The simulation of the collisional and irradiation processes on the object runs for $4 \times 10^{9} \mathrm{yr}$ with time steps of $10^{4} \mathrm{yr}$, and the canonical set of parameters used is listed in Table 1.

\section{Results}

A typical result of the simulation is shown in Fig. 3, where it is possible to observe that, any time the target receive a collision, the crystalline water ice fraction $(\eta)$ on the surface grows, while in the period between collisions the irradiation process transform the ice to its amorphous variety reducing the value of $\eta$ on the surface.

Since the simulations always begin with all the ice in an amorphous state $(\eta=0.0)$, to avoid any effect from the $\eta$ values 
Table 1. Canonical set of parameters used in the simulation.

\begin{tabular}{ll}
\hline \hline Target radius $\left(r_{\mathrm{t}}\right):$ & $8 \times 10^{7} \mathrm{~cm}$ \\
Target mean density $\left(\rho_{\mathrm{t}}\right):$ & $2.25 \mathrm{~g} \mathrm{~cm}^{-3}$ \\
Water ice density $\left(\rho_{\text {ice }}\right):$ & $1.00 \mathrm{~g} \mathrm{~cm}^{-3}$ \\
Rock density $\left(\rho_{\mathrm{s}}\right):$ & $3.50 \mathrm{~g} \mathrm{~cm}^{-3}$ \\
Projectile mean density $\left(\rho_{\mathrm{p}}\right):$ & $2.5 \mathrm{~g} \mathrm{~cm}^{-3}$ \\
Original mixed material initial depth $\left(h_{\text {mix }}\right):$ & $0 \mathrm{~cm}$ \\
Water ice fraction in original material $\left(f_{\text {ice }}\right):$ & 0.5 \\
Initial fraction of crystalline water ice $(\eta):$ & 0.0 \\
Target mean collisional velocity $\left(\left\langle V_{\text {col }}\right\rangle\right):$ & $2.48 \pm 0.86 \mathrm{~km} \mathrm{~s}^{-1}$ \\
Target mean intrinsic collision probability $(\langle P i\rangle):$ & $1.47 \pm 0.02 \times 10^{-22} \mathrm{~km}^{-2} \mathrm{yr}^{-1}$ \\
Time for total amorphization $\left(T_{\text {amorph }}\right):$ & $1 \times 10^{9} \mathrm{yr}$ \\
Thickness of layer with spectral information $\left(h_{\text {spec }}\right):$ & $200 \mu \mathrm{m}$ \\
Crater depth/diameter ratio $(\mu):$ & 0.2 \\
Energy fraction to kinetic energy of the fragments $\left(\delta_{\mathrm{k}}\right):$ & 0.2 \\
Energy fraction to thermal process $\left(\delta_{\mathrm{T}}\right):$ & 0.1 \\
Minimum thickness for global effect $\left(h_{\text {dep }}\right):$ & $0.01 \mu \mathrm{m}$ \\
Total simulation time $\left(T_{\text {sim }}\right):$ & $4 \times 10^{9} \mathrm{yr}$ \\
Simulation time step $\left(\Delta_{T}\right):$ & $1 \times 10^{4} \mathrm{yr}$ \\
Size distribution: & \\
$K_{0}$, & $4.11 \times 10^{26}$ \\
$q_{0}, q_{1}$ & $4.20,3.50-2.00$ \\
$r_{1}$ & $20-50 \mathrm{~km}$ \\
\hline
\end{tabular}

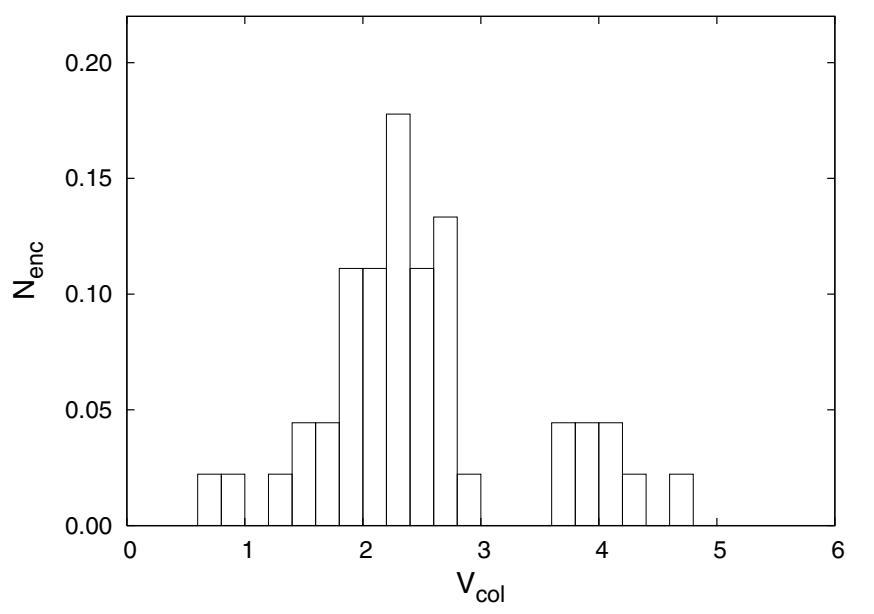

Fig. 2. Collision velocity distribution for (136108) $2003 E L_{61}$ obtained with the method of Marzari et al. (1996). The ordinate axis is the fraction of the total number of encounters between the target and a projectile.

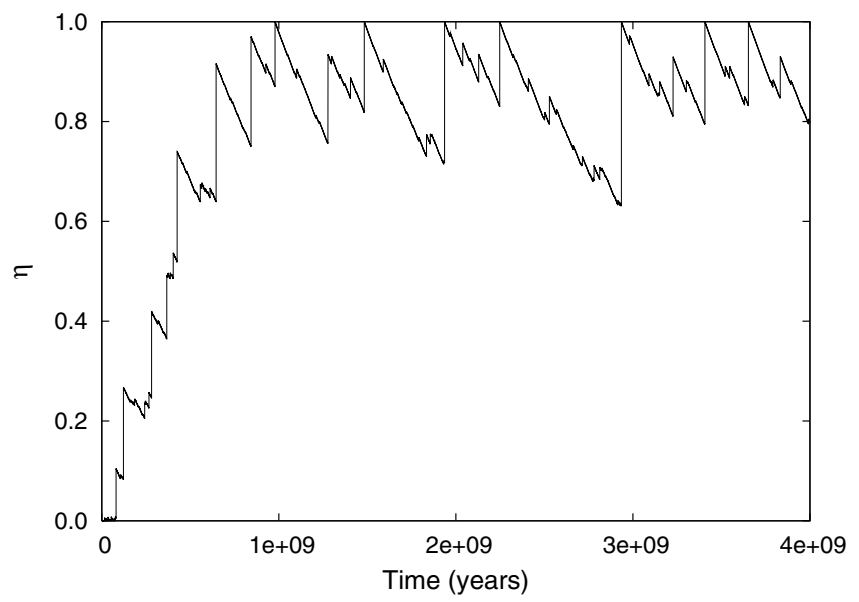

Fig. 3. Fraction of crystalline water ice obtained on the surface of (136108) $2003 E L_{61}$ for a break radius $40 \mathrm{~km}$ and a small end size distribution exponent $q_{1}=2.8$. obtained during the initial stabilization period of the simulation the mean value of the crystalline water ice fraction was calculated by using the results obtained only after the first $10^{9} \mathrm{yr}$.

To find the best values of $q_{1}$ and $r_{1}$, we arbitrarily fixed the value of $r_{1}$ and study how $\eta$ varies in function of $q_{1}$. We tested several value combinations for different parameters but the results obtained always change only slightly in comparison with those provided by the canonical set. The most significant parameters are $h_{\text {spec }}, h_{\mathrm{glob}}$, and $\delta_{T}$, which control the spectral information and the amount of crystallized water ice deposited on the surface after a collision, but still in these cases the results do not show a substantial change. For example, the mean value of $\eta$ for the canonical set of parameters and for a similar set but with $h_{\text {spec }}=100 \mu \mathrm{m}, h_{\text {dep }}=0.001 \mu \mathrm{m}$, or $\delta_{T}=0.5$ are shown in Figs. 4-6, respectively. The $r_{1}=50 \mathrm{~km}$ and $40 \mathrm{~km}$ cases are always characterized by a fast fall to $\eta=0.0$ for $q_{1}<2.2-2.3$ produced when the collisional resurfacing stops due to the short number of projectiles available in these size distributions, while for the $r_{1}=30 \mathrm{~km}$ and $20 \mathrm{~km}$ cases, the number of small projectiles is enough to support a collisional resurfacing process and the simulations also provided useful results for $q_{1}<2.2-2.3$.

Since the amorphization rate of irradiated crystalline water ice depends on the temperature, it is important to consider in the simulations variations in the temperature at which the amorphization starts to deviate from its $100 \%$ efficiency at low-temperature. Strazzulla et al. (1992) reported amorphization above or around $90 \%$ of the original crystalline ice at temperatures below $55 \mathrm{~K}$, Moore \& Hudson (1992) found that amorphization is complete for temperature below $46 \mathrm{~K}$, and Mastrapa $\&$ Brown (2006) find that, for a temperature equal to or below $40 \mathrm{~K}$, amorphization is complete, whereas above $50 \mathrm{~K}$ some crystalline character remains in the spectra. In any case, our model is not affected by such an effect, mainly because the surface temperature of(136108) $2003 E L_{61}$ is most probably below $40 \mathrm{~K}$ since Merlin et al. (2007) estimated a mean surface temperature of about $30 \mathrm{~K}$ for this body, and using the peak position of the $1.65 \mu \mathrm{m}$ band, they estimated an upper limit of $40 \mathrm{~K}$. Thus, the temperature at which we should consider the irradiation for (136108) $2003 E L_{61}$ is $\approx 30 \mathrm{~K}$ and, in any case, below $\approx 40 \mathrm{~K}$. 

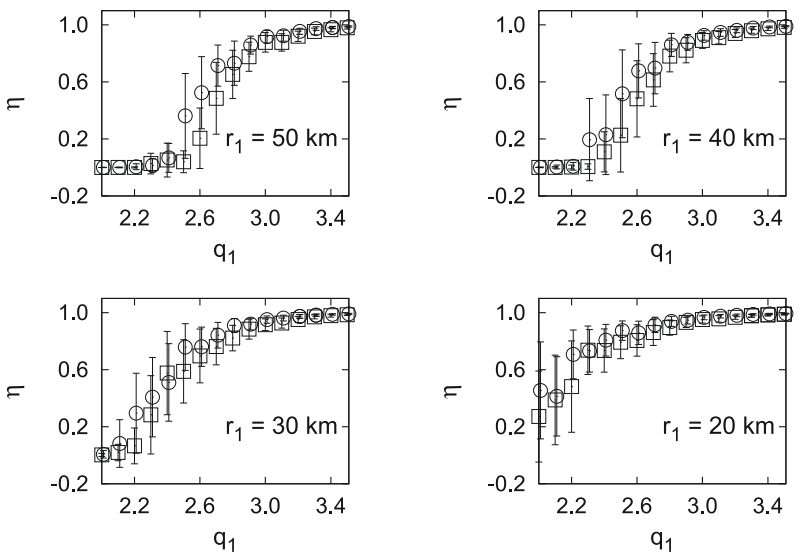

Fig. 4. Mean value for the fraction of crystalline water ice on the surface of (136108) $2003 E L_{61}$ for different smal-end size distribution exponents and break radius. The results for the canonical set of parameters are indicated by squares and those for a similar set but with $h_{\text {spec }}=100 \mu \mathrm{m}$ by circles. The error bars indicate the range of variations observed during the last $3 \times 10^{9} \mathrm{yr}$ of the simulation.
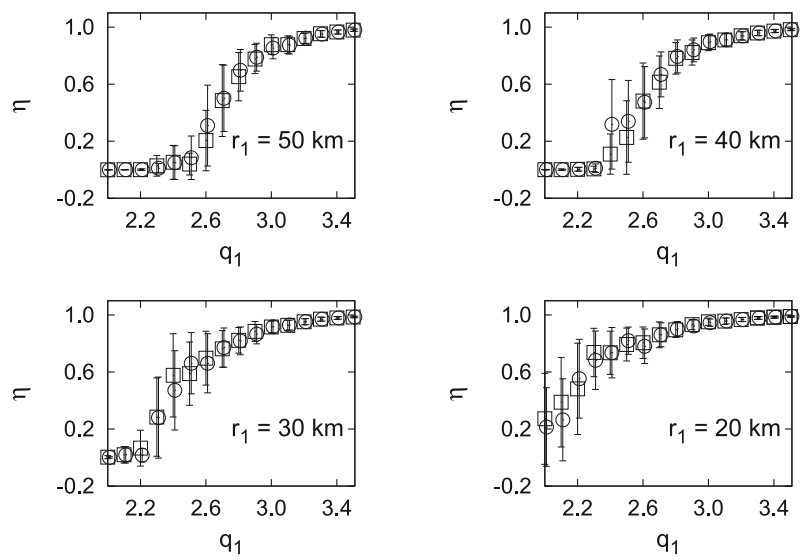

Fig. 5. Mean value for the fraction of crystalline water ice on the surface of (136108) $2003 E L_{61}$ for different small-end size distribution exponents and break radius. The results for the canonical set of parameters are indicated by squares and those for a similar set but with $h_{\text {dep }}=0.001 \mu \mathrm{m}$ by circles. The error bars indicate the range of variations observed during the last $3 \times 10^{9} \mathrm{yr}$ of the simulation.

Nevertheless, we also tested our model assuming that a $15 \%$ fraction of crystalline ice can survive after irradiation (Fig. 7). We find that, still in this case, the results obtained change only slightly in comparison with what is provided by the canonical set of parameters used.

The timescale for total amorphization of crystalline ice used in the model for an object at an heliocentric distance of $40 \mathrm{AU}$ is based on laboratory results obtained by Leto \& Baratta (2003) and Strazzulla et al. (2003) and could be in error by a certain amount. Then, we also tested our model using total amorphization times of $0.8 \times 10^{9} \mathrm{yr}$ (Fig. 8) and $1.2 \times 10^{9} \mathrm{yr}$ (Fig. 9), obtaining similar results in both cases to those provided by the canonical set of parameters.

The results for $r_{1}=20 \mathrm{~km}$ to $50 \mathrm{~km}$ using the canonical set of parameters are shown in Fig. 10. Taking the results of Pinilla-Alonso et al. (2009) for (136108) $2003 E L_{61}$ into account, we are looking for $\eta \approx 0.5$, so the best-fitted values for the small-end size distribution exponent obtained with these simulations are $q_{1}=2.65-2.75$ for $r_{1}=50 \mathrm{~km}, q_{1}=2.55-2.65$ for
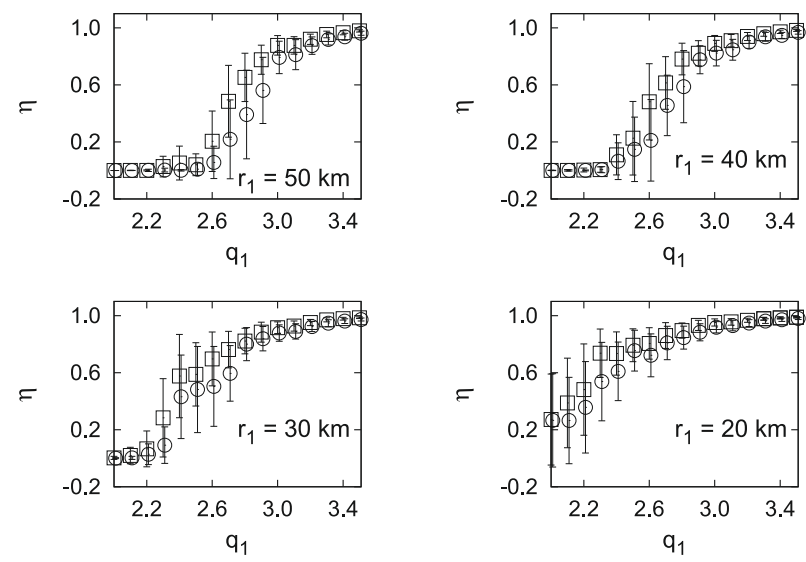

Fig. 6. Mean value for the fraction of crystalline water ice on the surface of (136108) $2003 E L_{61}$ for different small-end size distribution exponents and break radius. The results for the canonical set of parameters are indicated by squares and those for a similar set but with $\delta_{T}=0.05$ by circles. The error bars indicate the range of variations observed during the last $3 \times 10^{9} \mathrm{yr}$ of the simulation.
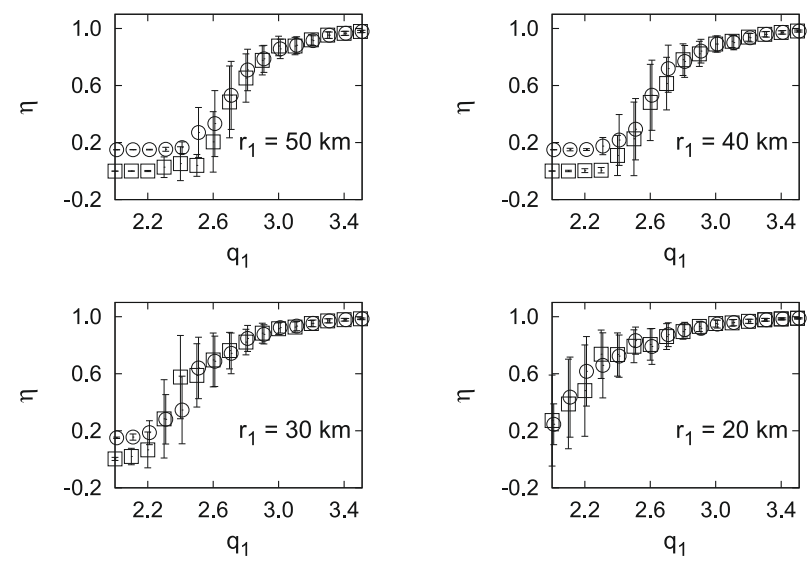

Fig. 7. Mean value for the fraction of crystalline water ice on the surface of (136108) $2003 E L_{61}$ for different smal-end size distribution exponents and break radius. The results for the canonical set of parameters are indicated by squares and those for a similar set but assuming that a $15 \%$ fraction of crystalline ice can survive after irradiation by circles. The error bars indicate the range of variations observed during the last $3 \times 10^{9} \mathrm{yr}$ of the simulation.

$r_{1}=40 \mathrm{~km}, q_{1}=2.35-2.45$ for $r_{1}=30 \mathrm{~km}$, and $q_{1}=2.2-2.3$ for $r_{1}=20 \mathrm{~km}$.

\section{Discussion}

The best way to compare these results with the observations is to calculate the cumulative luminosity function of the $\mathrm{TNb}$ population

$\log \Sigma\left(<m_{R}\right)=\alpha\left(m_{R}-m_{R_{0}}\right)$,

where $m_{R}$ is the red magnitude, $m_{R_{0}}$ the red magnitude at which one would expect to have 1 object per square degree in the sky, and $\alpha$ the slope of the cumulative luminosity function. The exponent of the size distribution is related with the slope of the cumulative luminosity function by $q=5 \alpha+1$ (Irwin et al. 1995). The red magnitudes are computed with

$m_{R}=m_{R \odot}-2.5 \log \left[\frac{p_{R} \Phi\left(\alpha^{\prime}\right) r^{2}}{2.25 \times 10^{16} R^{2} \Delta^{2}}\right]$ 

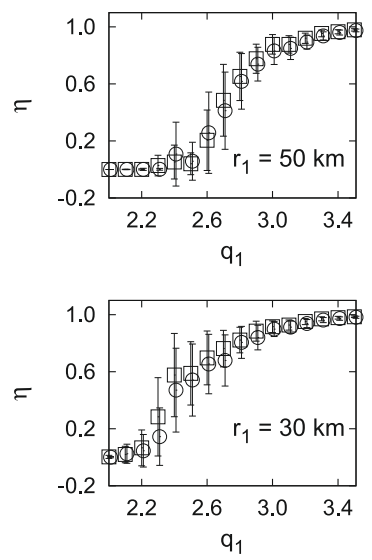
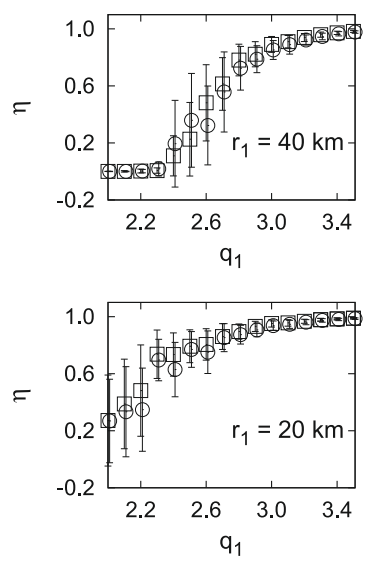

Fig. 8. Mean value for the fraction of crystalline water ice on the surface of (136108) $2003 E L_{61}$ for different small-end size distribution exponents and break radius. The results for the canonical set of parameters are indicated by squares and those for a similar set but assuming that the time needed to produce a total amorphization is $0.8 \times 10^{9} \mathrm{yr}$ by circles. The error bars indicate the range of variations observed during the last $3 \times 10^{9} \mathrm{yr}$ of the simulation.
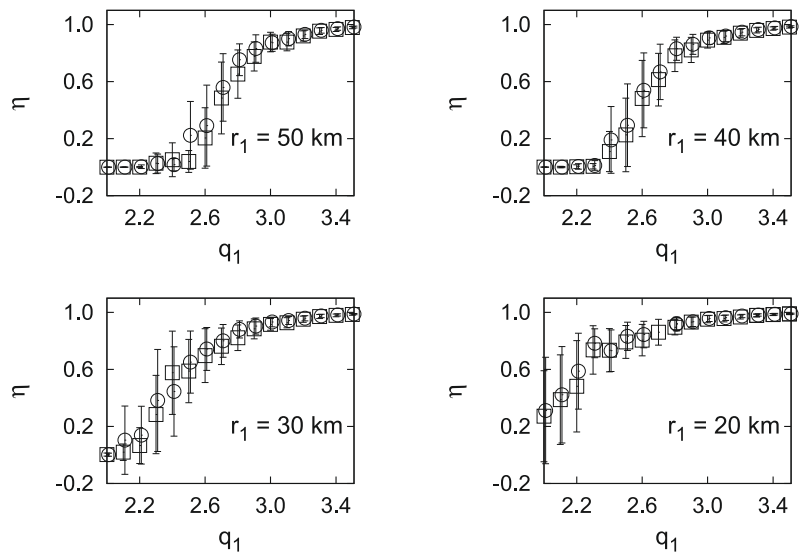

Fig. 9. Mean value for the fraction of crystalline water ice on the surface of (136108) $2003 E L_{61}$ for different small-end size distribution exponents and break radius. The results for the canonical set of parameters are indicated by squares and those for a similar set but assuming that the time needed to produce a total amorphization is $1.2 \times 10^{9} \mathrm{yr}$ by circles. The error bars indicate the range of variations observed during the last $3 \times 10^{9} \mathrm{yr}$ of the simulation.

where $m_{R \odot}=-27.1$ is the apparent red magnitude of the Sun, $\alpha^{\prime}$ the phase angle, $\Phi\left(\alpha^{\prime}\right)$ the Bowell et al. (1989) phase function, $p_{R}$ the geometric red albedo, $r$ the object radius in kilometers, and $R$ and $\Delta$ the heliocentric and geocentric distances in astronomical units, respectively. For this work, we assume $p_{R}=0.04$, $\Phi\left(\alpha^{\prime}\right)=1\left(\alpha^{\prime} \sim 0^{\circ}\right)$, and $R=40$ AU.

In Fig. 11 the cumulative luminosity functions obtained from the simulation are compared with the results of several TNO surveys (Jewitt \& Luu 1995; Irwin et al. 1995; Jewitt et al. 1996; Luu \& Jewitt 1998; Jewitt et al. 1998; Gladman et al. 1998; Chiang \& Brown 1999; Trujillo et al. 2001a,b; Gladman et al. 2001; Bernstein et al. 2004; Petit et al. 2006; Fraser et al. 2008). The observations at $m_{R}<25$ are well-fitted by the luminosity function proposed by Trujillo et al. (2001b) $(\alpha=0.66)$ and Fraser et al. (2008) $(\alpha=0.65)$, but for fainter magnitudes the observations seem to follow a luminosity function with a different slope like those we found in this paper.

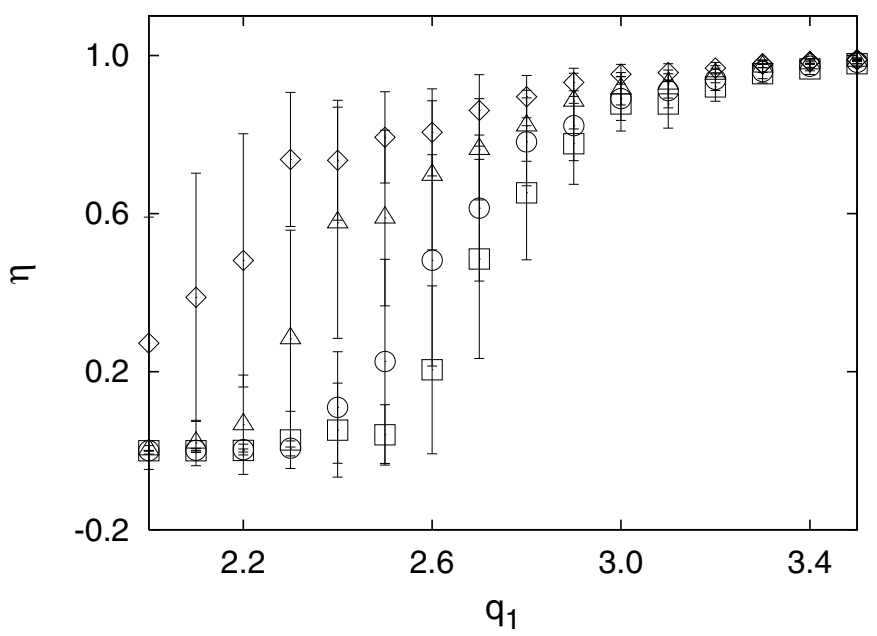

Fig. 10. Mean value for the fraction of crystalline water ice on the surface of (136108) $2003 E L_{61}$ for different small-end size distribution exponents and break radius using the canonical set of parameters. The case of $r_{1}=50 \mathrm{~km}$ are indicated by squares, $r_{1}=40 \mathrm{~km}$ by circles, $r_{1}=30 \mathrm{~km}$ by triangles, and $r_{1}=20 \mathrm{~km}$ by diamonds. The error bars indicate the range of variations observed during the last $3 \times 10^{9} \mathrm{yr}$ of the simulation.

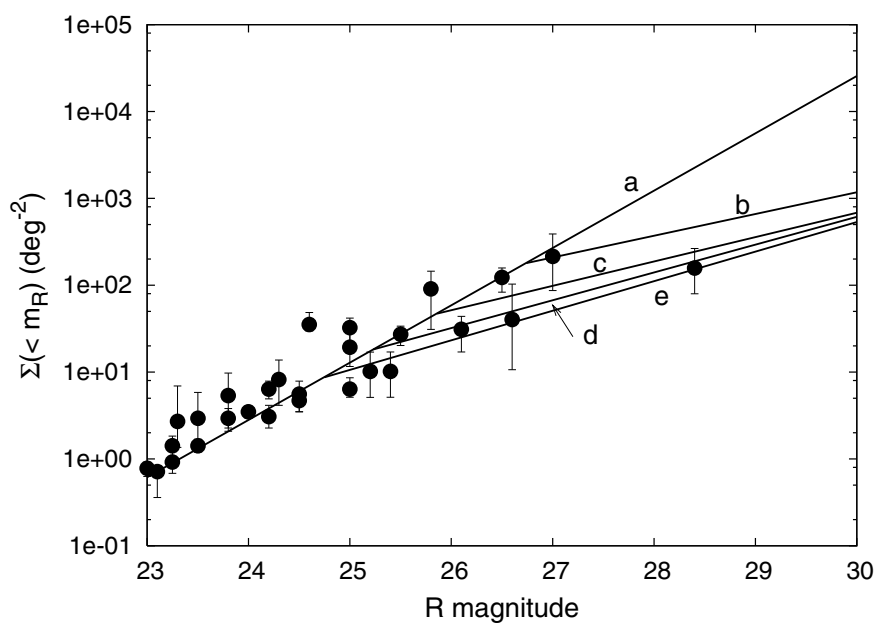

Fig. 11. Cumulative surface density of TNOs brighter than a given $R$ magnitude. The line indicated with "a" is the luminosity function for the size distribution of Trujillo et al. (2001b). The lines "b" to "e" are the luminosity functions for the calculated small end size distributions exponents $q_{1}=2.25,2.4,2.6$, and 2.7 for break radii of $r_{1}=20 \mathrm{~km}$, $30 \mathrm{~km}, 40 \mathrm{~km}$, and $50 \mathrm{~km}$, respectively.

Then, based on our simulations and the observations for $m_{R}>26$, we propose that the TNO size distribution changes its exponent from $q_{0}=4.2$ to $q_{1}=2.4 \pm 0.3$ at a break radius of $r_{1}=35 \pm 15 \mathrm{~km}\left(m_{R_{1}} \approx 25.5\right.$ for $\left.p_{R}=0.04\right)$. This result agrees very well with those obtained by Bernstein et al. (2004) $\left(q_{1}=2.6\right)$, Fuentes \& Holman (2008) $\left(q_{1}=2.5 \pm 1.0\right.$ and $\left.m_{R_{1}}=24.3_{-0.1}^{+0.8}\right)$, Fraser \& Kavelaars $(2008)\left(m_{R_{1}}=26.0_{-1.8}^{+0.7}\right)$, and Fraser \& Kavelaars (2009) $\left(q_{1}=1.9\right.$ and $\left.m_{R_{1}}=25.8\right)$.

It is important to note that, due to the constraint about the thickness limit of the crystalline ice layer deposited on the surface of the target after a collision $\left(h_{\mathrm{glob}}\right)$, the simulations are only sensitive to the effects of not very small projectiles $\left(r_{\mathrm{p}}>\right.$ 10-30 m), so it is possible that the exponent of the size distribution change again for these very small objects approaching a Donhanyi size distribution $(q=3.5)$. 


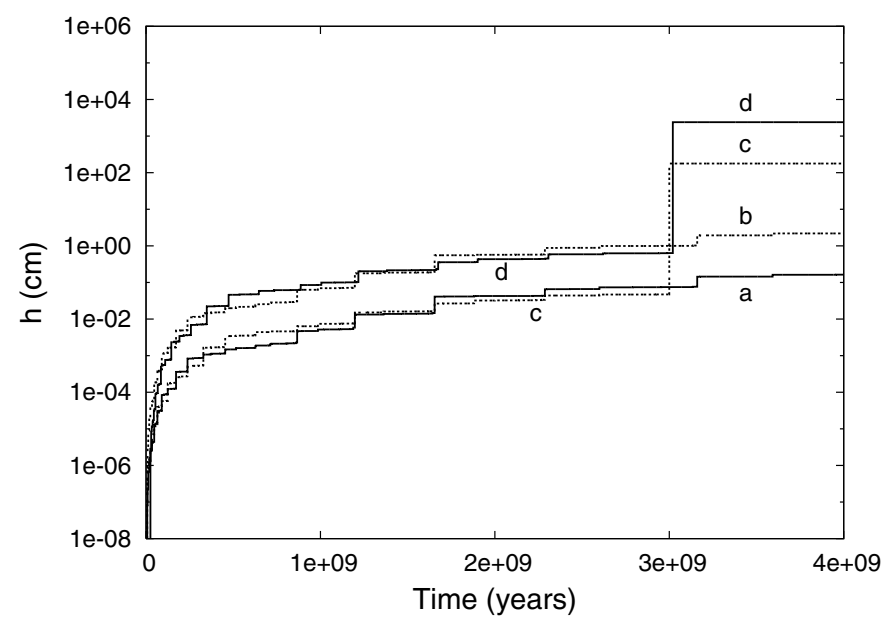

Fig. 12. Thickness of the external ice crust and the depth where the original material composition is present during the simulation with and without a collision. The lines "a" and "b" are the ice layer thickness and the depth at which the original composition is found, respectively, while the lines "c" and "d" indicate the same values but include the effects of a collision of the target with a projectile of $r_{\mathrm{p}}=50 \mathrm{~km}$, at $3 \times 10^{9} \mathrm{yr}$ from the beginning of the simulation.

If this size distribution is applied to studying the collisional surface evolution of (136108) $2003 E L_{61}$, we find that after $4 \times 10^{9} \mathrm{yr}$ the thickness of the most external ice crust is $0.12 \mathrm{~cm}$, while the original composition is present at $1.61 \mathrm{~cm}$ or more below the surface (Fig. 12). This external ice layer is very thin because it was formed by ice excavated by collisions with relatively small projectiles (in general, $r_{\mathrm{p}} \leq 2-3 \mathrm{~km}$ ). If we include in the simulation a collisional event with a projectile of $r_{\mathrm{p}}=100 \mathrm{~km}$ after, say, $3 \times 10^{9} \mathrm{yr}$ of the beginning of the simulation, the final ice thickness is $1.43 \mathrm{~m}$ and the original composition appears at a depth of $191 \mathrm{~m}$ below the surface.

In this last case, the effect of the collision is to cover the whole surface with a crystalline ice layer produced by the impact $(\eta=1)$, but after a few $10^{8} \mathrm{yr}$, the competition between the collisional and irradiation processes takes control again and the mean value obtained for $\eta$ is indistinguishable from what is obtained without a collision with a large projectile. It is important to mention that Lacerda et al. (2008a,b) find color variations with the rotational phase produced by a dark red spot on the surface of (136108) $2003 E L_{61}$. This red spot could be the result of a collision that destroyed the most external ice layer and exposed the underneath redder material. In this case, the sublimated ice produced by the collision precipitates over the surface and again covers the target with a crystalline ice layer, but if the collision occurred recently the ice layer produced by this and subsequent collisions is very thin (less than $200 \mu \mathrm{m}$ ), which is not enough to mask any silicate or amorphous ice contribution to the spectrum.

Since the present mean value of $\eta$ for 2003 EL $_{61}$ is not high (Pinilla-Alonso et al. 2009), the collisional scenario proposed by Brown et al. (2007) for the origin of the family of 2003 EL $_{61}$ should not happen recently, which also agrees with recent calculations indicating that the large impact that most probably created the family should be primordial (Ragozzine \& Brown 2007).

\section{Conclusions}

Using the peculiar crystalline to amorphous water ice ratio observed for (136108) $2003 E L_{61}$ as constraint, we found a best fit to the TNO size distribution at small sizes and its break radius. Our simulations indicate that the size distribution changes its exponent from $q_{0}=4.2$ to $q_{1}=2.4 \pm 0.3$ at a break radius of $r_{1}=35 \pm 15 \mathrm{~km}$. This result is not affected by a collision with a large projectile that occurred by chance during the simulation time.

Since the simulations are not sensitive to the effects of very small projectiles $\left(r_{\mathrm{p}}<10-30 \mathrm{~m}\right)$, it is possible that the exponent of the size distribution for these very small objects changes again approaching a Donhanyi size distribution.

If this size distribution is applied to studying the collisional surface evolution of (136108) $2003 E L_{61}$, we find that the object must be covered by a thin ice crust of $\approx 0.12 \mathrm{~cm}$, while the original composition of the object is still present at $1.61 \mathrm{~cm}$ or more below the surface. If we include a collisional event with a large projectile in the simulation, the final ice thickness becomes $1.43 \mathrm{~m}$ and the original composition appears at a depth of $191 \mathrm{~m}$ below the surface.

In this last case, the effect of the collision is to produce an ice layer with $\eta=1$, but after a short time the mean value obtained for $\eta$ is indistinguishable from what is obtained without a collision with a large projectile.

Finally, using the method proposed by Marzari et al. (1996), we also found for (136108) $2003 E L_{61}$ its mean collision velocity, $\left\langle V_{\text {col }}\right\rangle=2.48 \pm 0.86 \mathrm{~km} \mathrm{~s}^{-1}$, and mean intrinsic collisional probability, $\left\langle P_{i}\right\rangle=1.47 \pm 0.02 \times 10^{-22} \mathrm{~km}^{-2} \mathrm{yr}^{-1}$.

Acknowledgements. We thank G. Strazzulla and O. Gomis for useful discussion. J.L. and N.P.-A. acknowledge funding from the National Plan of I+D+i Project AYA2008-06202-C03-02 of Spanish Ministery of Science and Innovation. R.G.H. gratefully acknowledges support from San Juan National University grant $21 / \mathrm{E}-847$.

\section{References}

Barkume, K. M., Brown, M. E., \& Schaller, E. L. 2006, ApJ, 640, L87 Bernstein, G. M., Trilling, D. E., Allen, R. L., et al. 2004, AJ, 28, 1364 Bowell, E., Hapke, B., Domingue, D., et al. 1989, ed. R. Binzel, T. Gehrels, \& M. Matthews, Asteroids II (Tucson: Univ. of Arizona Press), 524

Brown R., Cruikshank, D., \& Pendleton Y. 1999, ApJ, 519, L101

Brown, M. E., Barkume, K. M., Ragozinne, D., et al. 2007, Nature, 446, 294 Brunetto, R., \& Roush, T. L. 2008, A\&A, 481, 879

Brunetto, R., Barucci, M., Dotto, M. E., \& Strazzulla, G. 2006, ApJ, 644, 650 Brunetto, R., Roush, T. L., Marra, A. C., \& Orofino, V. 2007, Icarus, 191, 381 Chiang, E. I., \& Brown, M. E. 1999, AJ, 118, 1411

Davis, D. R., \& Farinella, P. 1996, Icarus, 125, 50 Davis, D. R., \& Farinella, P. 1997, Icarus, 125, 50 Dell'Oro, A., Marzari, F., Paolicchi, P., \& Vanzani, V. 2001, A\&A, 366, 1053 Dohnanyi, J. W. 1969, J. Geophys. Res., 74, 2531

Fernández, J. A., Gallardo, T., \& Brunini, A. 2002, Icarus, 159, 358

Fraser, W. C., \& Kavelaars, J. J. 2008, Icarus, 198, 452

Fraser, W. C., \& Kavelaars, J. J. 2009, AJ, 137, 72

Fraser, W. C., Kavelaars, J. J., Holman, M. J., et al. 2008, Icarus, 195, 827

Fuentes, C. I., \& Holman, M. J. 2008, AJ, 136, 83

Gil-Hutton, R. 1997, Planet. Space Sci., 45, 229

Gladman, B., Kavelaars, J. J., Nicholson, P. D., Loredo, T. J., \& Burns, J. A. 1998, AJ, 116, 2042

Gladman, B., Kavelaars, J. J., Petit, J.-M., et al. 2001, AJ, 122, 1051

Hapke, B. 1993, Theory of Reflectance and Emittance Spectroscopy (New York: Cambridge Univ. Press)

Holsapple, K. A. 1993, Annu. Rev. Earth Planet. Sci. 21, 333

Irwin, M., Tremaine, S., \& Zytkow, A. N. 1995, AJ, 110, 3082

Jewitt, D., \& Luu, J. 1995, AJ, 109, 1867

Jewitt, D., Luu, J., \& Chen, J. 1996, AJ, 112, 1225

Jewitt, D., Luu, J., \& Trujillo, C. 1998, AJ, 115, 2125

Johnson, R., Lanzerotti, L., \& Brown, W. 1984, Adv. Space Res., 4, 41

Kenyon, S. J. 2002, PASP, 114, 265

Kenyon, S. J., \& Bromley, B. C. 2004, AJ, 128, 1916

Kenyon, S. J., \& Windhorst, R. A. 2001, ApJ, 547, L69

Kouchi, A., \& Kuroda, T. 1990, Nature, 344, 134

Lacerda, P., Jewitt, D., \& Peixinho, N. 2008a, AJ, 135, 1749 
Lacerda, P., Jewitt, D., \& Peixinho, N. 2008b, AJ, 136, 1754

Leto, G., \& Baratta, G. A. 2003, A\&A, 397, 7

Licandro, J., di Fabrizio, L., Pinilla-ALonso, N., et al. 2006, A\&A, 457, 323

Luu, J., \& Jewitt, D. 1998, ApJ, 502, L91

Marzari, F., Scholl, H., \& Farinella, P. 1996, Icarus, 119, 192

Mastrapa, R. M. E., \& Brown, R. H. 2006, Icarus, 183, 207

Merlin, F., Guilbert, A., Dumas, C., et al. 2007, A\&A, 466, 1185

Moore, M. H., \& Hudson, R. L. 1992, ApJ, 401, 353

Moore, M., Donn, B., Khanna, R., \& A'Hearn, M. 1983, Icarus, 54, 388

Orosei, R., Coradini, A., De Sanctis, M. C., \& Federico, C. 2001, Adv. Space res., 28, 1563

Pan, M., \& Sari, R. 2005, Icarus, 173, 342

Petit, J.-M., Holman, M. J., Gladman, B. J., et al. 2006, MNRAS, 365, 429

Pinilla-Alonso, N., Licandro, J., Gil-Hutton, R., \& Brunetto, R. 2007, A\&A, 468, L25

Pinilla-Alonso, N., Brunetto, R., Licandro, J., et al. 2009, A\&A, in press

Ragozzine, D., \& Brown, M. E. 2007, AJ, 134, 2160
Rabinowitz, D. L., Barkume, K., Brown, M. E., et al. 2006, ApJ, 639, 1238

Schenk, P. M., Chapman, C., Moore, J., \& Zahnle, K. 2004, in Jupiter: Planet, Satellites, and Magnetosphere, ed. F. Bagenal, \& W. McKinnon (Cambridge: Cambridge Univ. Press), 427

Skorov, Yu. Y., Kömle, N. I., Keller, H. U., et al. 2001, Icarus, 153, 180

Stern, S. A. 2002, AJ, 124, 2297

Strazzulla, G., \& Johnson, R. 1991, in Comets in the Post-Halley era, ed. R. L. Newburn, Jr., M. Neugebauer, \& J. Rahe (Netherlands: Kluwer Academic Publishers), 243

Strazzulla, G., Baratta, G. A., Leto, G., \& Foti, G. 1992, Europhys. Lett., 18, 517 Strazzulla, G., Cooper, J. F., Christian, E. R., \& Johnson, R. E. 2003, C. R. Phys, 4,791

Sunshine, J. M., A'Hearn, M. F., Groussin, O., et al. 2006, Science, 311, 1453

Trujillo, C. A., Jewitt, D. C., \& Luu, J. X. 2001a, AJ, 122, 457

Trujillo, C. A., Luu, J. X., Bosh, A. S., \& Elliot, J. L. 2001b, AJ, 122, 2740

Trujillo, C. A., Brown, M., Barkume, K. M. et al. 2007, ApJ, 655, 1172

Zheng, W., Jewitt, D., \& Kaiser, R. I. 2008 [arXiV: 0801.2805] 